\title{
The Role of Color Doppler Ultrasonography and Magnetic Resonance in the Prenatal Diagnosis of Placenta Accrete
}

\author{
Hayam A. Abdellatif, Mona K. Alashwah, Mohamed S. Elzawawi
}

Department of Radio-diagnosis, Faculty of Medicine, Menoufia University, Egypt

*Correspondence author: Hayam Abdelmonsif Abdellatif, Mobile: (+20)1098057324, E-mail: hayam_drar@yahoo.com

\begin{abstract}
Background: Placenta accrete is a clinical and diagnostic challenge that is being with increasing frequency and may lead to catastrophic blood loss and related complications. Color doppler ultrasonography and magnetic resonance imaging are considered as specific imaging modalities and have very important role in the prenatal diagnosis of placenta accreta. Objectives: The aim of the current work was to evaluate the diagnostic value of color doppler ultrasonography and magnetic resonance imaging in the prenatal assessment of placenta accreta.

Patients and Methods: This study was conducted on 40 pregnant women (from 32 to 40 weeks of pregnancy) with placenta previa and presented with manifestations of suspected placenta accreta. Their age ranged from 19 to 40 years with mean age of 31.8 years. All cases were subjected to ultrasound examination, but MRI was done in 33 cases prior to elective delivery. Results: Cases diagnosed of having placenta accreta were 18 out of 40 cases (45\%). Among those 18 cases; placenta found to be accreta in 6 patients, increta in 7 patients and percreta in 5 patients. Our study showed high statistical significance between occurrence of Placenta accrete \& prior uterine surgeries as a risk factor. Overall accuracy of ultrasound was $88 \%$, while that of MRI was $85 \%$. Ultrasound showed better specificity (86\%) than that of MRI (81\%), but MRI showed better sensitivity (92\%) than that of ultrasound (89\%).

Conclusion: It could be concluded that both ultrasound and magnetic resonance have fairly good sensitivity and specificity for prenatal diagnosis of placenta accreta. Color doppler ultrasound remains the first primary screening imaging modality used to evaluate suspected placenta accreta and MRI is suggested as an alternative complementary tool to ultrasound in cases with inconclusive criteria.
\end{abstract}

Keywords: Accreta, Color Doppler, Placenta, Ultrasonography, Magnetic resonance.

\section{INTRODUCTION}

Placenta accreta is abnormal placental adherence or invasion of the myometrium or extra-uterine structures. It is one of the main causes of excessive postpartum hemorrhage. Placenta Accreta account for $33-50 \%$ of all emergency peri-partum hysterectomies (1). It is classified based on the depth of myometrial invasion. In placenta accreta vera, the mildest form, villi are attached to the myometrium but do not invade the muscle. In placenta increta, villi partially invade the myometrium. The most severe form is the placenta percreta, in which villi penetrate through the entire myometrial thickness or beyond the serosa ${ }^{(2)}$.

The most common risk factors are placenta previa, previous caesarean section and old maternal age. Placenta previa has increased nowadays parallel to the increasing cesarean delivery ${ }^{(3)}$. There is an increase has also been seen in vitro fertilization pregnancies. The two major diagnostic modalities used nowadays in prenatal diagnosis are ultrasound and magnetic resonance imaging. Ultrasound is always the first imaging modality used to evaluate suspected placenta accreta. This modality has a great popularity because it is widely available, patient friendly, non-invasive, and relatively inexpensive ${ }^{(4)}$. Although ultrasound remains the primary screening modality for the detection of abnormal placentation, MRI is a complementary technique that should be considered when ultrasound is equivocal or additional information is needed for example, in a patient with prior uterine surgery, instrumentation, or injury involving the posterior myometrium, sonographic visualization may be limited by fetal position or other factors. In such cases in which concern for possible placental invasion is raised, targeted magnetic resonance imaging might be of benefit ${ }^{(5)}$. The exact process is still unknown but is probably more complicated than just abnormal decidualization of a scarred area or lack of decidua in the lower uterine segment near the cervix. The consequences are often caesarean hysterectomy and the end of fertility, as well as increased rates of blood loss and transfusion, injury to surrounding organs, and increased rates of intensive care admission when compared with women who undergo caesarean section for placenta previa alone. Forewarning and preparation, including a multidisciplinary approach, do help to reduce morbidity ${ }^{(6)}$. The aim of the present study was to evaluate the diagnostic value of color doppler ultrasonography and magnetic resonance imaging in the prenatal assessment of placenta accreta.

\section{PATIENTS AND METHODS}

This study included a total of 40 pregnant women (from 32 to 40 weeks of pregnancy) with placenta previa and presented with manifestations of suspected placenta accreta, attending at Menoufia University Hospitals. Subject's age ranged from 19 to 40 years (mean age: 31.8 years). All cases were subjected to ultrasound examination. MRI was done in 33 cases prior to elective delivery. 


\section{Inclusion criteria:}

Pregnant women with placenta previa in the third trimester of pregnancy (from 32 to 40 weeks of pregnancy) implanted on the lower uterine segment with a previous cesarean delivery, or dilatation and curettage, and with or without painless vaginal bleeding, they were evaluated for the possibility of placenta accreta. Such cases were at high risk for placenta accreta regarding their clinical history of either one or all the following:

1. Maternal age $>35$ years.

2. Grand multiparity.

3. Previous uterine interventional procedures (e.g., cesarean sections, dilatation \& curettage and Myomectomy) in addition to the major co-existing factor which is placenta previa.

\section{Each patient in this study was subjected to:}

- Full history taking including past history of uterine surgery, such as prior cesarean section.

- Clinical examination.

- Informed consent was obtained before scanning.

- Ultrasound examination was performed using a 2D ultrasound system equipped with a $5 \mathrm{MHz}$ transabdominal transducer.

- MRI scan has been done after ultrasound examination in some patients.

- All of 40 cases were subjected to Ultrasound (US) and MRI was done in 33 cases prior to elective delivery.

\section{Ultrasound technique:}

For each patient, the whole placenta was scanned using both gray-scale ultrasound and color flow mapping. In women with previous caesarean section, there was an attention to the uterine scar which was carefully localized and evaluated for possible invasive placenta. Patients were examined in supine position with a sufficient bladder volume to clearly visualize the serosa- bladder interface. Using longitudinal view: to assess the depth of placental tissue, its vascularity and its relationship to the bladder wall, axial imaging is used to assess the extent of invasion and color Doppler study.

All ultrasound scans were done using a Philips HD 11 XE ultrasound machines using a $5 \mathrm{MHz}$ transabdominal convex transducer.

\section{On gray-scale ultrasound imaging:}

Criteria suggestive of placenta accreta (including its variants, placenta increta and placenta percreta):

- Placenta previa.

- Multiple vascular lacunae within the placental mass, giving a "Swiss cheese" appearance.

- Complete loss of hypoechoic space between placenta and myome $\neg$ trium on ultrasound examination.

- Irregular retro placental sonolucent zone.

- Retro placental myometrial thickness of less than 1 $\mathrm{mm}$.
- Bulging" of the placental-myometrial tissues into adjacent spaces (e.g., into the urinary bladder).

- Color Doppler criteria:

- Turbulent flow of lacunae.

- Increased vascularity of the bladder-serosal interface.

- Blood vessels bridging the uterine-placental or myometrial-bladder interface.

- Markedly dilated vessels over the peripheral subplacental region.

\section{MRI technique:}

Magnetic resonance imaging (MRI) scans have been done for 33 patients with ultrasonographic criteria suggestive of placenta accreta to provide more accurate diagnosis of the placental location and to check for radiological signs of placental invasion. All MRI examinations were performed on a 1.5-T unit (TOSHIBA EXCELART VANTAGE, Japan) super conducting magnet using body array coils. Cases were imaged in the supine position with their feet placed first into the scanner to minimize feeling of claustrophobia. The bladder was partially full for better visualization of the myometrial/bladder interface. The entire examination time was 25- 30 minutes. Intravenous injection of gadolinium was not done.

The maternal pelvis was scanned using fast spin echo (FSE) sequences in assessing placental invasion to the myometrium or beyond using the following protocol:

1. Axial T1W, T2W FSE (FOV $426 \mathrm{~mm} ; 8.8 \mathrm{~mm}$ slice thick sections; matrix 192 x 384; scan duration 1:30 $\min$ ).

2. Sagittal and coronal T1W FSE (FOV $380 \mathrm{~mm} ; 10$ $\mathrm{mm}$ thick sections; matrix $256 \times 380$; scan duration for coronal $1.40 \mathrm{~min}$, for sagittal $4.46 \mathrm{~min}$ ).

3. Sagittal and coronal T2W FSE (FOV $380 \mathrm{~mm} ; 10$ mm thick sections; matrix 192 x 384; scan duration for coronal $2 \mathrm{~min}$, for sagittal $4 \mathrm{~min}$ ).

4. Sagittal T1W fat sat (FOV 380; $10 \mathrm{~mm}$ slice thickness; matrix 288; scan duration 4min).

5. Sagittal (Gradient) T2* (FOV 380; $10 \mathrm{~mm}$ slice thickness; Matrix 192 x 352; scan duration 6 min).

\section{MRI criteria of placenta accreta:}

- Placenta previa.

- Heterogeneous signal within the placenta.

- Dark intraplacental bands on T2-weighted images.

- Focal bulging of the placenta causing a contour deformity of the uterus.

- Focal interruption of the myometrium.

- Direct visualization of invasion of adjacent structures (usually the bladder).

- Tenting of the bladder.

\section{Ethical consent:}

An approval of the study was obtained from Menoufia University academic and ethical committee. Every patient signed an informed 
written consent for acceptance of the operation. This work has been carried out in accordance with The Code of Ethics of the World Medical Association (Declaration of Helsinki) for studies involving humans.

\section{Statistical analysis}

Data were collected and entered to the computer using SPSS (Statistical Package for Social Science) program for statistical analysis.

Descriptive statistics: Quantitative data were shown as mean, standard deviation $( \pm \mathrm{SD})$ and range. Qualitative data were expressed as frequency and percent at $95 \%$ confidence interval. Analytical statistics: Chi-square test (X2) was used to measure association between qualitative variables. $P$ (probability) value considered to be of statistical significance if it is less than 0.05 . Accuracy was represented using the terms sensitivity, specificity, +ve predictive value, -ve predictive value, and overall accuracy. The significance of the results was assessed in the form of P-value that was differentiated into: -Non-significant when P-value >0.05. - Significant when $\mathrm{P}$-value $\leq 0.05$. Highly significant when $\mathrm{P}$-value $\leq 0.01$.

\section{RESULTS}

The study included 40 pregnant females with placenta previa at high risk of the presence of placenta accrete. Among the 40 cases, placenta previa was found to be complete previa in 27 cases $(67.5 \%)$ \& marginalis in 13 cases (32.5\%), as shown in Chart (1) and history of prior uterine surgery as (cesarean section and/ or D\& C) was elicited in $95 \%$ of cases 38 out of 40 , as shown in Chart (2). Their age ranged from 19 to 40 years with mean age of 31.8 years, gestational age ranged from 32 to 39 week with mean of 34 week.
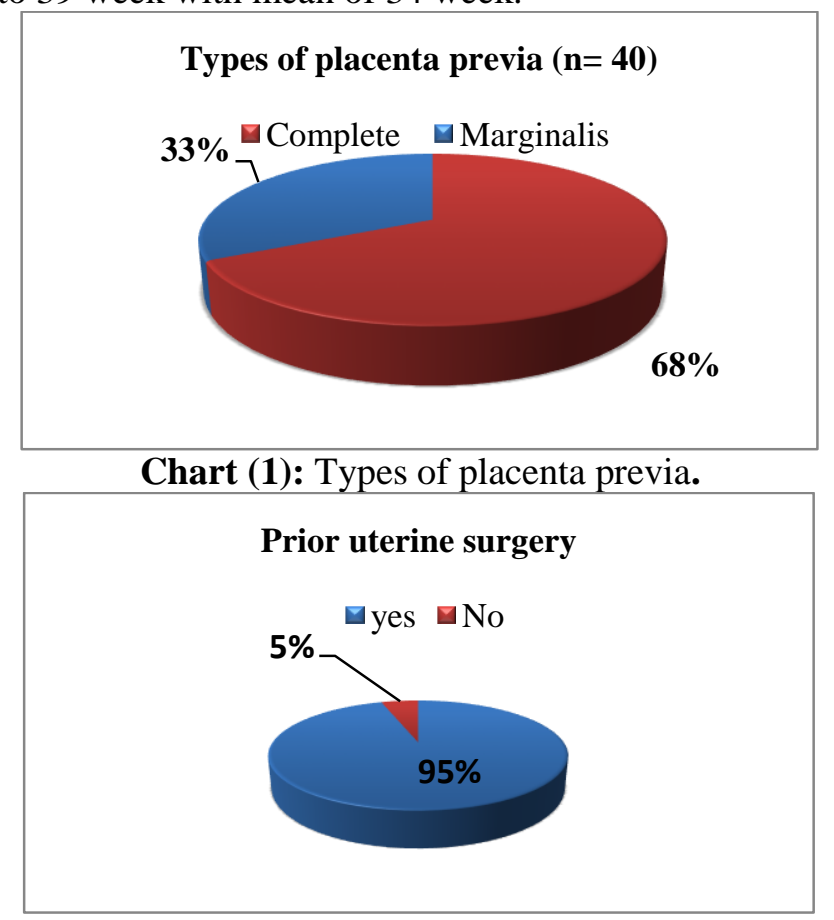

Chart (2): History of prior uterine surgery.
Overall accuracy of ultrasound for diagnosis of placenta accreta was $88 \%$, while overall accuracy of MRI was $85 \%$ (Table1).

Table (1): The accuracy of U/S and MRI imaging in the diagnosis of placenta accrete.

\begin{tabular}{|l|c|c|c|c|c|c|}
\hline & Sensitivity & Specificity & $\begin{array}{c}\text { P- } \\
\text { value }\end{array}$ & Accuracy & PPV & NPP \\
\hline $\begin{array}{c}\text { Ultra- } \\
\text { sound }\end{array}$ & $89 \%$ & $86 \%$ & $\begin{array}{c}<0.00 \\
1 \mathrm{HS}\end{array}$ & $88 \%$ & $\begin{array}{c}84 \\
\%\end{array}$ & $\begin{array}{c}90 \\
\%\end{array}$ \\
\hline MRI & $92 \%$ & $81 \%$ & $\begin{array}{c}0.001 \\
\mathrm{~S}\end{array}$ & $85 \%$ & $\begin{array}{c}73 \\
\%\end{array}$ & $\begin{array}{c}94 \\
\%\end{array}$ \\
\hline
\end{tabular}

$\mathrm{PPV}=$ positive predictive value $\mathrm{NPP}=$ Negative Predictive value

Table (2): Clinical diagnosis at delivery of the studied patients.

\begin{tabular}{|l|l|c|c|}
\hline \multicolumn{2}{|c|}{} & \multicolumn{2}{c|}{ Studied patients (n=40) } \\
\cline { 3 - 4 } \multicolumn{2}{|c|}{} & NO & \% \\
\hline \multirow{2}{*}{$\begin{array}{l}\text { Clinical } \\
\text { diagnosis } \\
\text { at delivery }\end{array}$} & Accreta & 6 & $15 \%$ \\
\cline { 2 - 4 } & Increta & 7 & $17.5 \%$ \\
\cline { 2 - 4 } & Percreta & 5 & $12.5 \%$ \\
\cline { 2 - 4 } & Previa & 22 & $55 \%$ \\
\hline
\end{tabular}

Thirteen cases out of eighteen (72.2\%) proved to be accreta have had cesarean hysterectomy and one of those 13 done partial cystectomy with cesarean hysterectomy, while the remaining 27 cases had cesarean section.

This shows a highly statistically significant relationship between hysterectomy and placenta accreta in our study of $p$ value $=0.008$, as shown in Chart (3).

Relation between plcenta accreta and hysterectomy $(\mathrm{n}=18)$

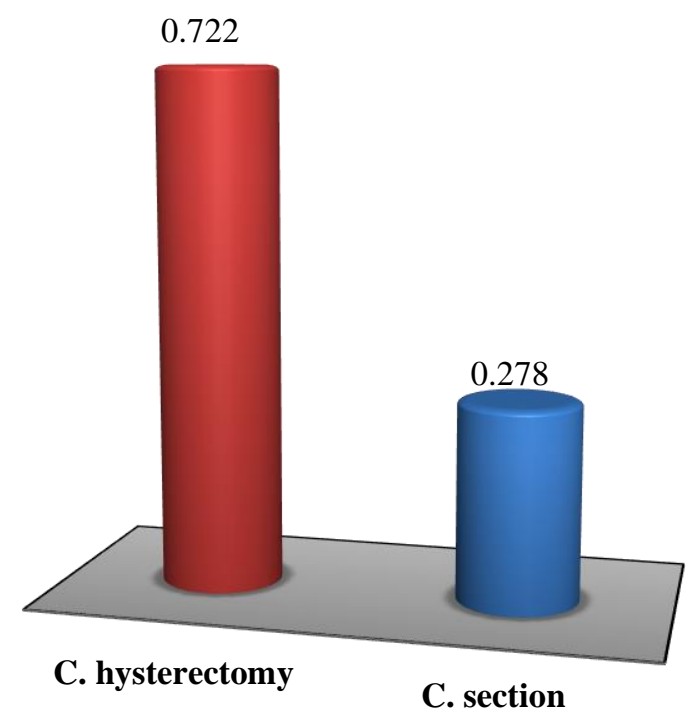

Chart (3): Relation between placenta accreta and hysterectomy. 
CASE (1):
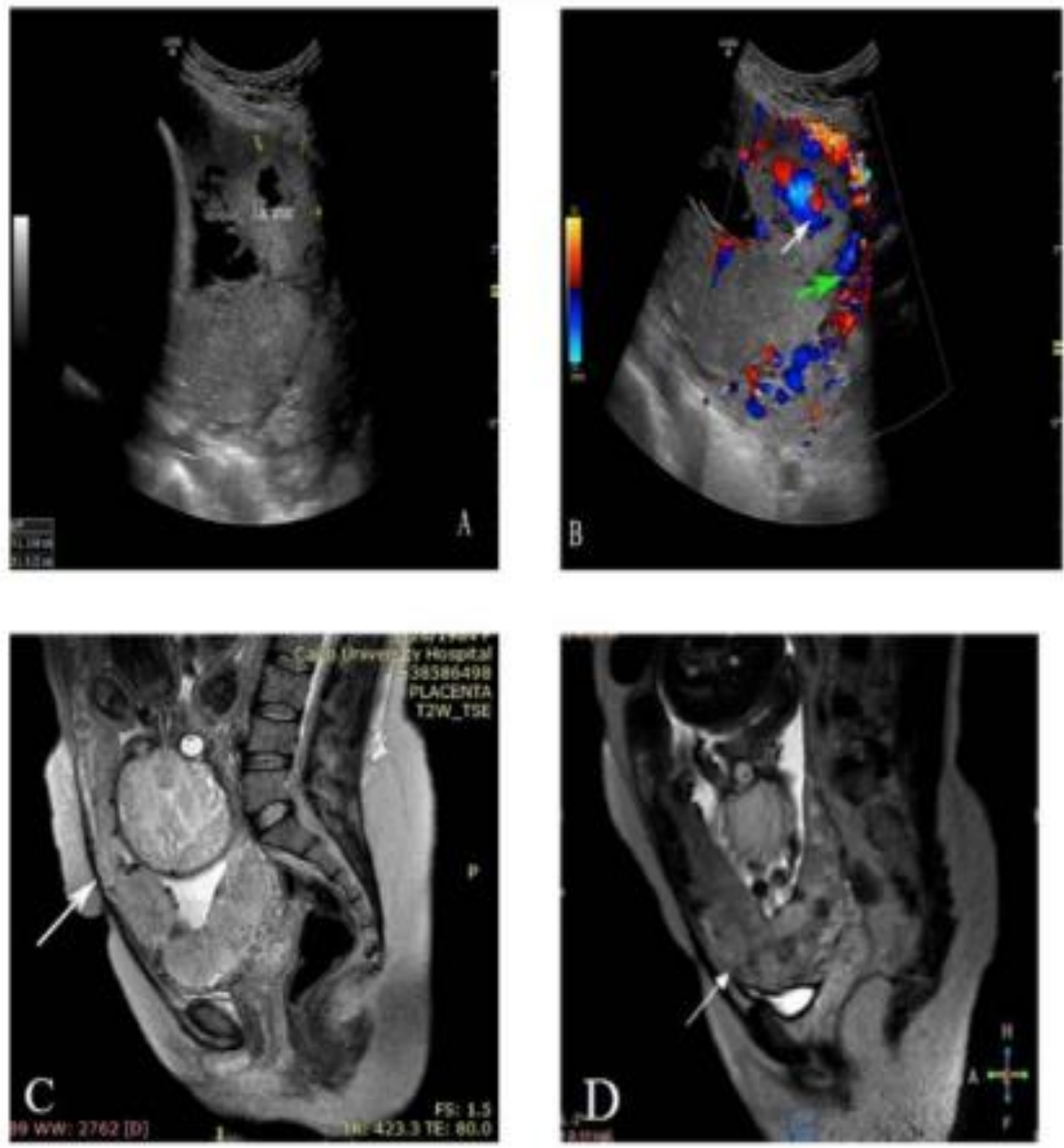

Female patient, 33years, at 36 weeks presented by vaginal bleeding. She had history of 1 previous cesarean section. Color Doppler \& U/S (A-B): Trans-abdominal gray scale (image A) US shows bilobed placenta previa complete centralis with lacunae and thinned out myometrium (cursors), by color Doppler (B), turbulent flow within the lacunae (white arrow) and uterine serosa hypervascularity (green arrow).

MRI findings (C-D): MRI T2W sagittal (C) shows heterogenous placenta with lacunae, focal (white arrow) and sagittal T2W (D) shows evidence of infiltration of inner layer of the myometrium in the lower uterine segment anteriorly (Arrow). The case was placenta increta and cesarean hysterectomy was done. 
CASE (2):
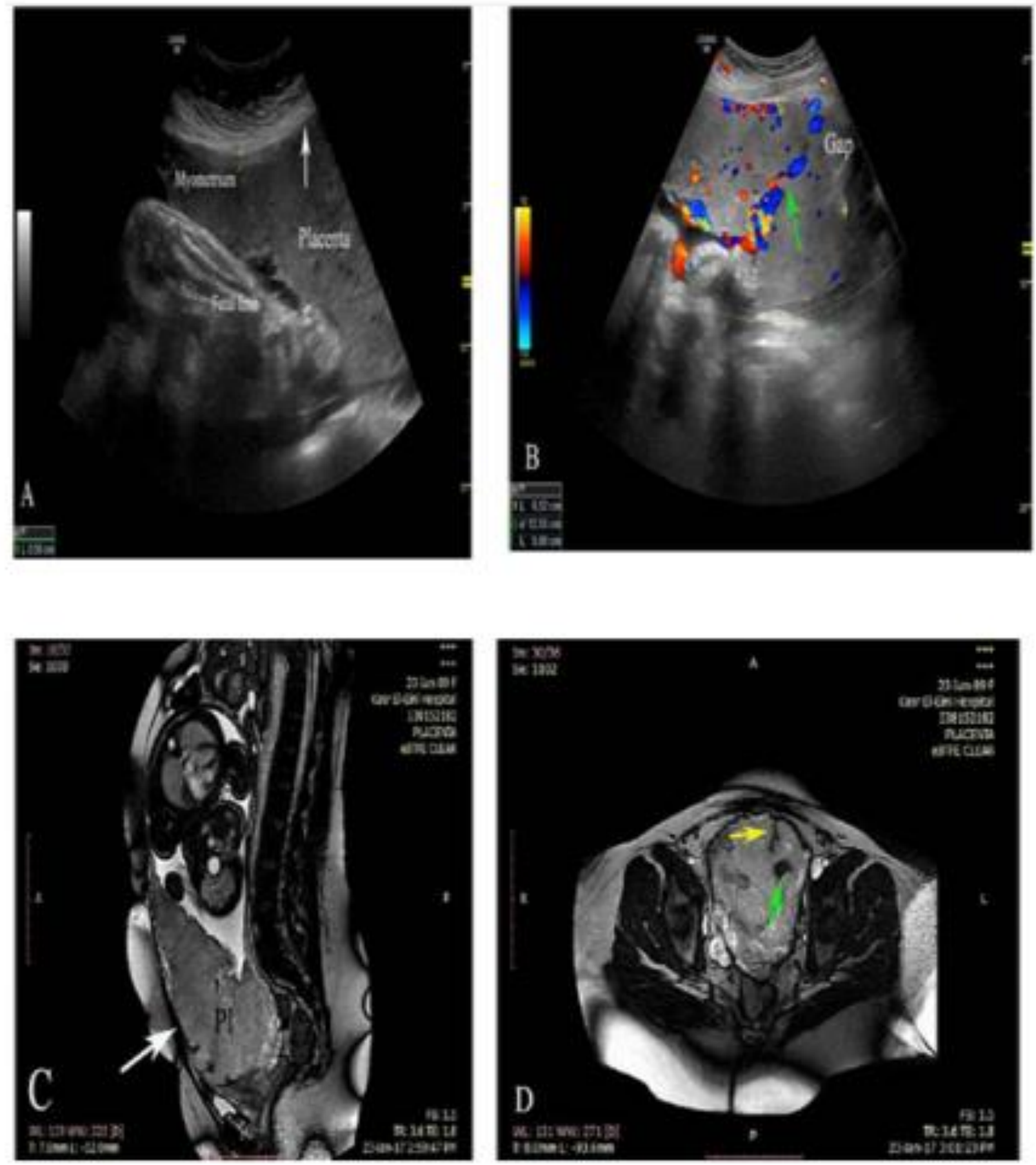

Female patient, 30 years old, 37 weeks presented by vaginal bleeding. She had history of previous 2 cesarean section.

Color Doppler \& U/S (A-B): Shows Placenta previa with vascular lacunae and lost myometrium (white arrow) by gray scale u/s (A). Focal gap in retro-placental blood flow (cursors) and crossing vessel (green arrow) between both placental surfaces seen by color Doppler (B).

MRI (C-D): Sagittal T2W (C) shows heterogenous placenta with macro-lobulated outlines and thinned out myometrium (white arrow) opposite to cesarean scar and preserved vesico-uterine serosa fat plane and Axial T2W (D) shows dark band (yellow arrow) and lacunae (green arrow). The case was placenta increta and cesarean hysterectomy was done. 
CASE (3)
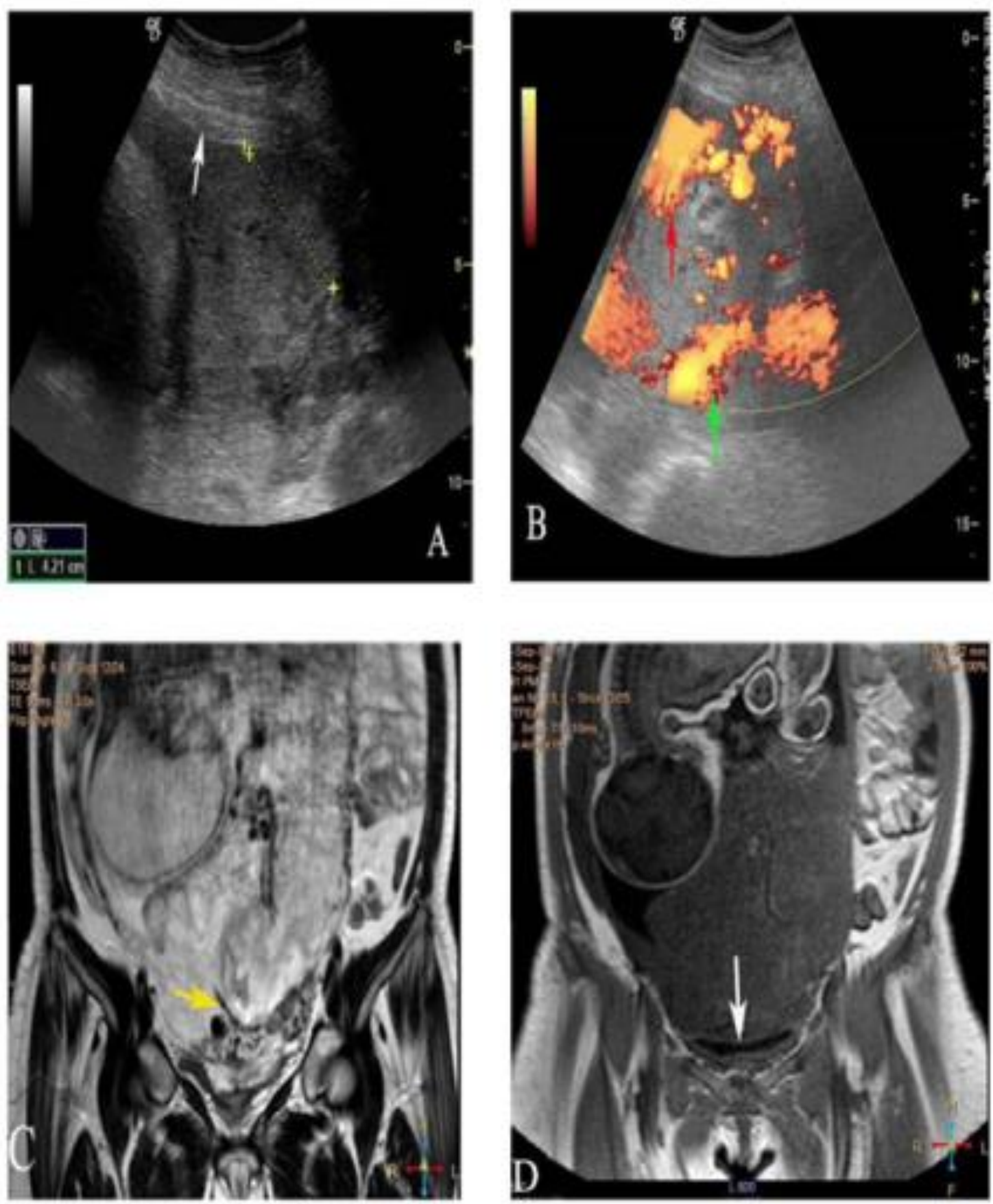

Female patient, 35 years old, 37 weeks presented by vaginal bleeding. She had history of previous 2 cesarean section and $1 \mathrm{D} \& \mathrm{C}$.

Color Doppler \& U/S (A-B): Shows placenta previa complete centralis (A) focal loss of myometrium (cursors) (white arrow points to normal myometrium thickness. (B) color Doppler shows hypervascularity within placental lacunae (red arrow) and peripheral sub-placental hypervascularity (green arrow). MRI (C-D): T2W sagittal (C) shows heterogenous placenta with nodular outline. Suspicion of percreta by the presence of vessels crossing between the bladder \& myometrium in the utero-vesical space (yellow arrow). T1W coronal (D) shows obliteration of fat plane between bladder and uterine serosa (white arrow). The case was placenta percreta and cesarean hysterectomy with partial cystectomy was done. 
CASE (4):
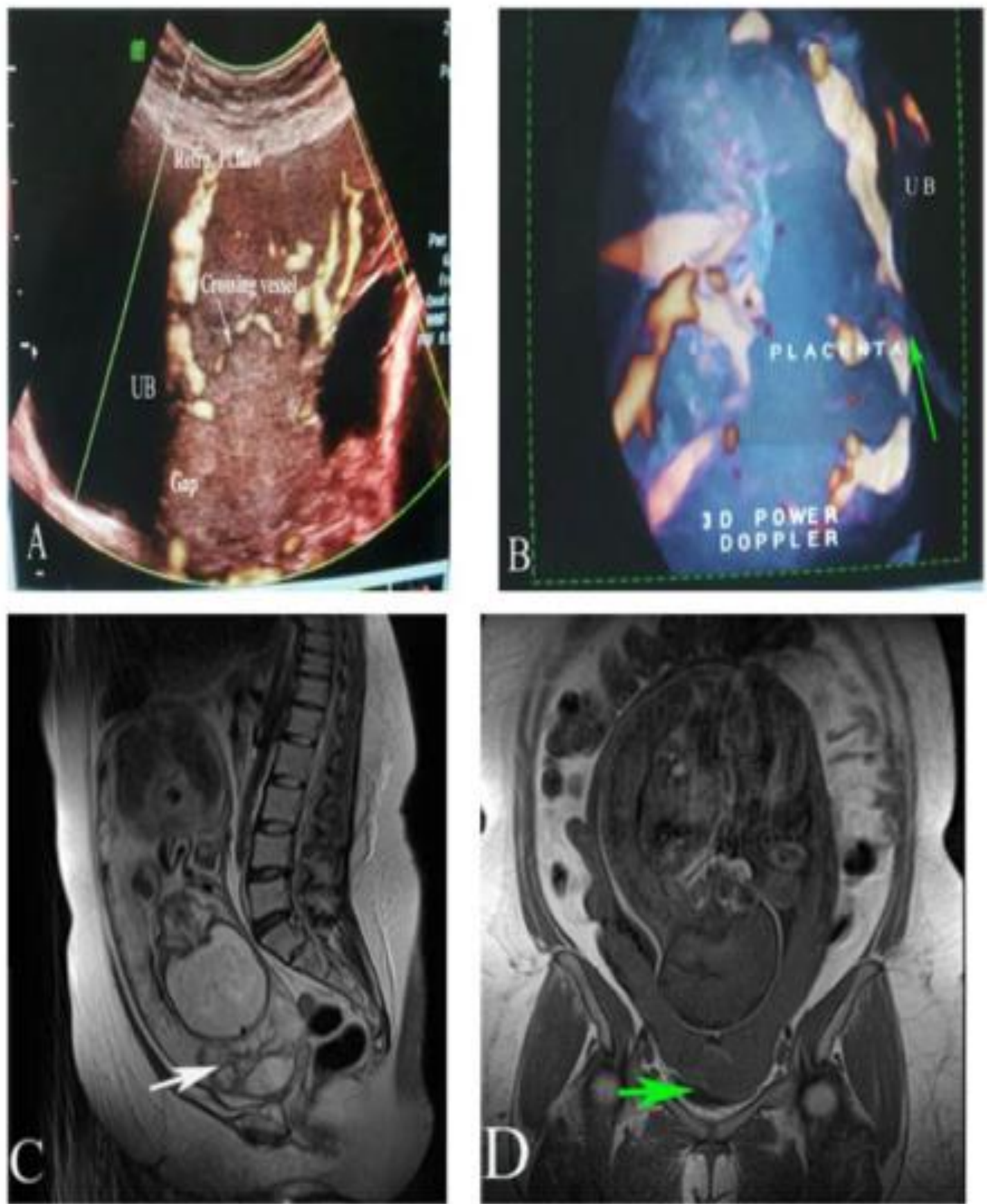

Female patient, 31 years, at 37weeks presented by vaginal bleeding. She had history of 2 cesarean section and 1 D\& C.

Color Doppler \& U/S (A-B): Color Doppler us (A) shows focal gap in retro-placental flow, lacunar flow and aberrant vessel crossing placenta (white arrow). Focal obliteration of vesico- uterine serosa space (green arrow) by 3D power Doppler (B). MRI (C-D): T2W sagittal (C) shows heterogenous placenta with dark bands (white arrow) and thinned out myometrium. T1W coronal (D) shows preserved of vesico- uterine fat plane (green arrow). The case was placenta increta and cesarean hysterectomy was done. 
CASE (5):
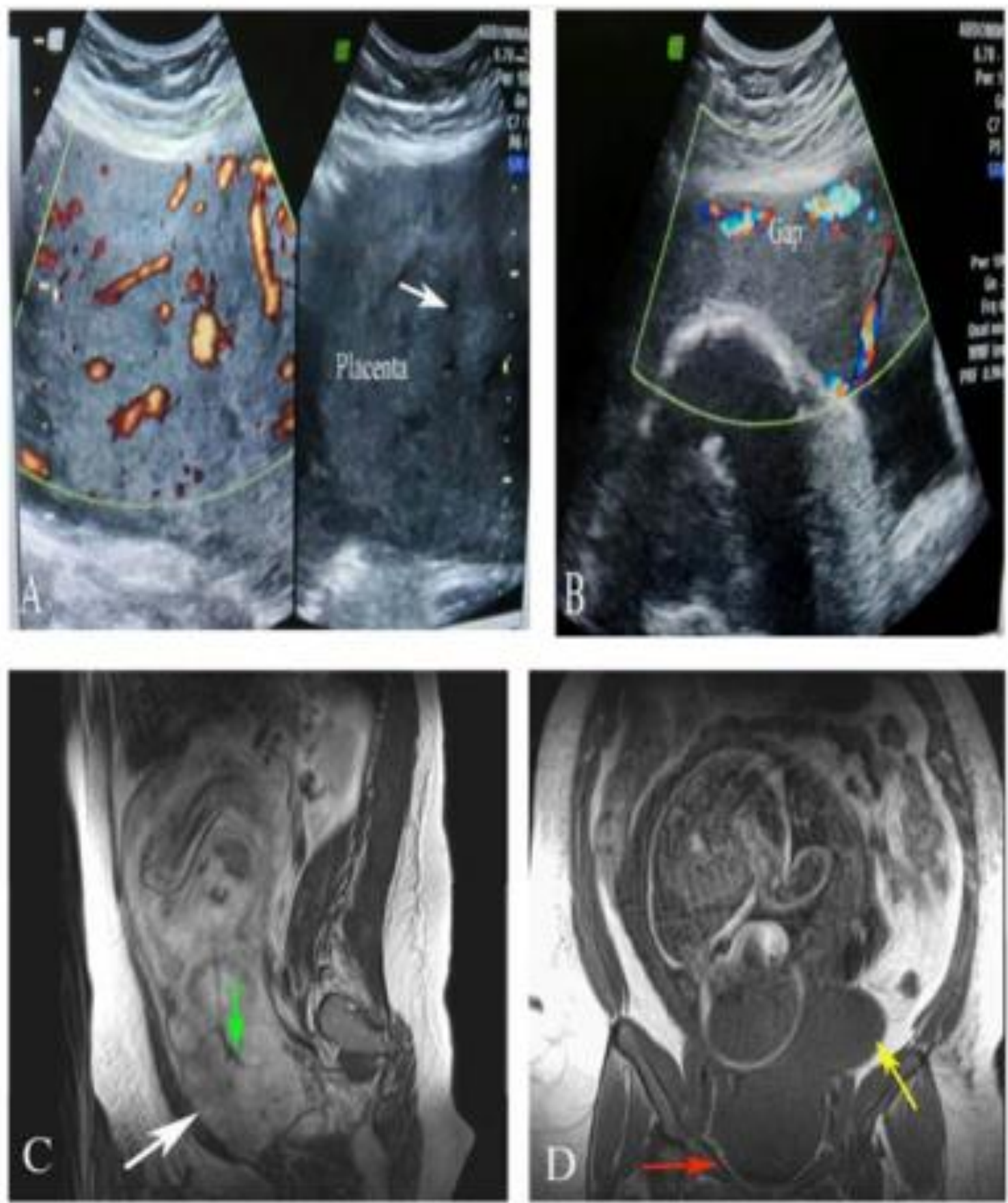

Female patient, 37 years old at 38 weeks. She had history of previous 3 cesarean section. Diagnosed to have Placenta Previa during routine antenatal examination.

Color Doppler \& U/S (A-B): Gray scale \& color Doppler U/S (A) shows placenta complete previa with hypo- echoic vascular lacunae (white arrow). By color Doppler (B) focal gap in retro- placental flow was seen. MRI (C-D): T2W sagittal (C) shows heterogenous placenta with thinned out myometrium (white arrow) and dark intra placental band (green arrow). T1W coronal (D) shows Focal bulge of placenta (yellow arrow) with preserved vesico-uterine fat plane (red arrow). The case was Placenta increta and cesarean hysterectomy was done. 


\section{DISCUSSION}

Placenta accreta is a significant cause of maternal morbidity and mortality and is now the most common reason for emergent postpartum hysterectomy. Placenta previa and a prior history of cesarean section are the most significant risk factors for it. In fact, that, as indications for cesarean delivery is steadily increasing, including cesarean delivery on maternal request, the incidence of placenta accreta will continue to increase ${ }^{(7)}$.

Early recognition of the risk factors is the key for early antenatal diagnosis and better management of placenta accreta. Prior cesarean section, prior dilatation and curettage or any uterine surgeries with presence of placenta previa are the main risk factors and has been found in about $95 \%$ of placenta accreta patients ${ }^{(\mathbf{8})}$. Ultrasound is always the first imaging modality used to evaluate suspected placenta accreta. This modality has a great popularity because it is widely available, noninvasive, non-ionizing radiation and relatively inexpensive ${ }^{(9)}$. However, some authors have said that ultrasound could be limited in assessment depth of invasion in patients with suspected placenta accreta. Others pointed that it lacks the adequate resolution to diagnose accreta in posterior placenta or the obesity of the patient which presents a barrier through the scan ${ }^{(10)}$.

MRI has been suggested as an alternative to US. It has better soft tissue contrast, thereby providing better resolution and allowing accurate measurement of depth of invasion. It has shown to be beneficial in some cases when ultrasound findings are equivocal or nondiagnostic. This include patients with single positive criterion to be placenta accreta especially if the criterion is of low specificity as obliteration of the clear space, patients with placenta previa posterior \& caesarean section scar, patients with placenta previa and previous accreta $^{\left({ }^{(10)} \text {. }\right.}$

Placenta previa findings that were considered suggestive of accreta on color Doppler US included: (1) loss of the retroplacental hypoechoic clear zone, (2) loss of the bladder wall-uterine interface, (3) presence of placental lacunae (4) presence of hypervascularity of the interface between the uterine serosa and the bladder wall on color Doppler imaging. Regarding MRI; findings of placenta accreta: placenta previa, uterine bulging, heterogeneous signal intensity within placenta, dark intraplacental bands on T2 weighted images, abnormal disorganized placental vascularity, focal interruptions in the myometrial wall, tenting of the bladder, Direct visualization of invasion of pelvic structures by the placental tissue ${ }^{(11)}$.

Mansour et al. ${ }^{(11)}$ reveled that cases diagnosed of having placenta accreta as reported by cesarean delivery data were $42.8 \%(n=15 / 35)$, all of them had previous uterine surgeries. Matching between US and MRI findings took place in $75 \%(n=15 / 20)$ of the negative cases and in $80 \%(\mathrm{n}=12 / 15)$ of the positive cases of placenta accreta in the studied cohort. So they concluded that MRI hand in hand with ultrasound is important for accurate diagnosis. Another study done by Satija et al. ${ }^{(12)}$ concluded that there were no statistical differences in sensitivity and specificity between USG and MRI except for posteriorly located placenta and useful in patients with ambiguous USG findings. Presence of placenta previa, placental lacunae with turbulent flow, loss of retroplacental clear space, and gap in the retroplacental blood flow were the most common findings on CDUS. Heterogeneous signal intensity within placenta, dark intraplacental bands on T2W images, and abnormal disorganized placental vascularity were the most common findings on MRI. Sensitivity and specificity of MR was estimated without the use of gadolinium, which is the approach most suitable for gravid patients since the European Medicines Agency warns against the use of gadolinium before the first year of life, this study found that as placenta accreta was presented in eight patients. USG correctly identified the presence of placenta accreta in seven out of eight patients ( $87.5 \%$ sensitivity) and the absence of placenta accreta in 19 out of 22 patients (86.4\% specificity). MRI correctly identified the presence of placenta accreta in 6 out of 8 patients ( $75.0 \%$ sensitivity) and absence of placenta accreta in 17 out of 22 patients (77.3\% specificity).

Masselli et al. ${ }^{(13)}$ reported that MR and US Doppler showed no statistically difference in identifying patients with placental invasion while MRI was statistically better than US Doppler in evaluating the depth of placenta infiltration. In conclusion, the study confirmed that pelvic US is highly reliable to diagnose or exclude the presence of placental invasion and found MRI to be an excellent tool for the staging evaluation of placenta accreta. Dwyer et al. (14) concluded that both sonography and MRI have fairly good sensitivity for prenatal diagnosis of placenta accreta; however, specificity does not appear to be as good as reported in other studies. In the case of inconclusive findings with one imaging modality, the other modality may be useful for clarifying the diagnosis.

Chou et al. ${ }^{(15)}$ used the following color Doppler criteria; placental lacunar flow, interruption of retroplacental blood flow, uterine serosa- bladder interphase hypervascularity \& peripherally sub-placental hypervascularity. According to the preceding criteria, 16/ 80 diagnosed as accreta but only 14 cases histopathologically proved of being accreta. Two cases had false positive color Doppler; this was mistaken for uterine serosa- bladder hypervascularity caused by bladder varcies. Of the 64 cases with negative results, three had accreta; two of them were with posterior placenta previa and last one with lateral placenta previa. 
This study recommended that color Doppler ultrasound has a great role in antenatal diagnosis of placenta accreta as it highlights abnormal areas of hypervascularity with dilated blood vessels within the placenta and uterine tissues.

Warshak et al. ${ }^{(16)}$ concluded that a two-stage protocol for evaluating women at high risk for placenta accreta, which uses ultrasonography first, and then MRI for cases with inconclusive ultrasound features, will optimize diagnostic accuracy. This study used gadolinium contrast in MRI examination as they believed that the use of gadolinium-based contrast enhancement adds to the specificity of MRI in the diagnosis of placenta accreta because it more clearly delineates the outer placental surface relative to the myometrium and eliminates the confusion between heterogeneous signals thought to be within the placenta from those caused by maternal blood vessels. The use of gadolinium in pregnancy is still controversial, as it crosses placenta, enters the fetal circulation, and is excreted by the fetal kidney, so its fetal effects are unknown and since the kidney is considered immature in children younger than 1 year, the European Medicines Agency warns that gadolinium should be used with caution in this age group (12). Another comparable study done by Elhawary et al. (17) suggested that US and MRI were useful in the diagnosis of placenta accreta with lacunae and an abnormal color Doppler imaging pattern are the most helpful findings. MR imaging is most clearly indicated when US findings are ambiguous or there is a posterior placenta.

\section{CONCLUSION}

It could be concluded that ultrasonography remains the diagnostic standard, and routine US examination affords an ideal opportunity to screen for the placental invasion. Antenatal sonographic imaging can be complemented by magnetic resonance imaging in equivocal cases to distinguish those women at high risk of placenta accreta.

\section{REFERENCES}

1. Mar W, Berggruen S, Atueyi U et al. (2015): Ultrasound imaging of placenta accreta with MR correlation. Ultrasound Quarterly, 31(1): 23-33.

2. Saraví P, Patiño N, Juana M et al. (2014): Doppler Ultrasound in the diagnosis of placenta percreta: our experience. Rev Argent Radiol., 78(3): 149-55.

3. Comstock C, Bronsteen R (2014): The antenatal diagnosis of placenta accreta. BJOG: An International Journal of Obstetrics \& Gynaecology, 121(2): 171-82.
4. Esh-Broder E, Ariel I, Abas-Bashir N et al. (2011): Placenta accreta is associated with IVF pregnancies: a retrospective chart review. BJOG., 118: 1084-9.

5. Leyendecker J, DuBose $\mathrm{M}$, Hosseinzadeh $\mathrm{K}$ et al. (2012): MRI of pregnancy-related issues: abnormal placentation. American Journal of Roentgenology, 198 (2): $311-320$.

6. Esakoff T, Sparks T, Kaima A et al. (2011): Diagnosis and morbidity of placenta accreta. Ultrasound in Obstetrics \& Gynecology, 37(3): 324-28.

7. Warshak C, Ramos G, Eskander R et al. (2010): Effect of predelivery diagnosis in 99 consecutive cases of placenta accreta. Obstetrics \& Gynecology, 115(1): 65-9.

8. Eller A, Porter T, Soisson P et al. (2009): Optimal management strategies for placenta accreta. BJOG: An International Journal of Obstetrics \& Gynaecology, 116(5): 648-54.

9. Levine D, Barnes P, Edelman R (2005): Obstetric MR Imaging. Radiology, 211(3): 609-17.

10. Lam G, Kuller J, McMahon M (2002): Use of magnetic resonance imaging and ultrasound in the antenatal diagnosis of placenta accreta. Journal of the Society for Gynecologic Investigation, 9(1): 37-40.

11. Mansour S, Elkhyat W (2011): Placenta previa accreta: Do we need MR imaging? The Egyptian Journal of Radiology and Nuclear Medicine, 42:433-42.

12. Satija B, Kumar S, Wadhwa L et al. (2015): Utility of ultrasound and magnetic resonance imaging in prenatal diagnosis of placenta accreta: A prospective study. The Indian Journal of Radiology \& Imaging, 25(4): 464-72.

13. Masselli G, Brunelli R, Casciani E et al. (2008): Magnetic resonance imaging in the evaluation of placental adhesive disorders: correlation with color Doppler ultrasound. European Radiology, 18(6): $1292-$ 9.

14. Dwyer B, Belogolovkin V, Tran L et al. (2008): Prenatal diagnosis of placenta accreta sonography or magnetic resonance imaging? J Ultrasound Med., 27:1275-81.

15. Chou M, Ho E, Lee Y (2000): Prenatal diagnosis of placenta previa accreta by transabdominal color Doppler ultrasound. Ultrasound in Obstetrics \& Gynecology, 15(1): 28-35.

16. Warshak C, Eskander R, Hull A et al. (2006): Accuracy of ultrasonography and magnetic resonance imaging in the diagnosis of placenta accreta. Obstetrics \& Gynecology, 108(3): 573-81.

17. Elhawary T, Dabees N, Youssef M (2013): Diagnostic value of ultrasonography and magnetic resonance imaging in pregnant women at risk for placenta accreta. The Journal of Maternal-Fetal \& Neonatal Medicine, 26(14): 1443-9. 